\title{
CAPACIDADE FUNCIONAL E QUALIDADE DE VIDA EM IDOSOS COM OSTEOARTROSE NO MUNICÍPIO DE COARI - AM
}

\author{
Nádia Gomes B. Santos Acadêmica do curso de Fisioterapia do Instituto de \\ Saúde e Biotecnologia, Universidade Federal do \\ Amazonas. \\ Esmeraldino Monteiro Figueiredo Neto \\ Fisioterapeuta. Professor Assistente do curso de \\ Fisioterapia da Faculdade de Educação Física e \\ Fisioterapia, Universidade Federal do Amazonas . \\ Guilherme P. Tinoco Arêas Fisioterapeuta. Professor Assistente do curso de \\ Fisioterapia do Instituto de Saúde e Biotecnologia, \\ Universidade Federal do Amazonas. \\ Fernando Zanela da Silva Arêas Fisioterapeuta. Professor Assistente do curso de \\ Fisioterapia do Instituto de Saúde e Biotecnologia, \\ Universidade Federal do Amazonas . \\ Hércules Ribeiro Leite Fisioterapeuta. Professor Assistente do curso de \\ Fisioterapia do Instituto de Saúde e Biotecnologia, \\ Universidade Federal do Amazonas . \\ Márcio Antônio Couto Ferreira \\ Fisioterapeuta. Professor Assistente do curso de \\ Fisioterapia do Instituto de Saúde e Biotecnologia, \\ Universidade Federal do Amazonas . \\ Renato Campos Freire Júnior Fisioterapeuta. Professor Assistente do curso de \\ Fisioterapia do Instituto de Saúde e Biotecnologia, \\ Universidade Federal do Amazonas .

\begin{abstract}
Resumo
Introdução: A osteoartrite $(\mathrm{OA})$ é uma doença crônico-degenerativa reumática com elevada prevalência entre idosos que gera incapacidade funcional e elevado impacto sobre a qualidade de vida do portador. Entretanto, diferenças regionais relacionadas aos hábitos de vida podem influenciar estes aspectos. Objetivo: Avaliar a qualidade de vida (QV) e a capacidade funcional (CF) de idosos com osteoartrose, na cidade de Coari, Amazonas. Metodologia: Foram utilizados dois questionários para a mensuração da CF e QV, o índice de Katz e o SF- 36 respectivamente. O coeficiente linear de Pearson foi adotado com medida de intensidade de associação entre as variáveis quantitativas. Resultados: Participaram da pesquisa 25 idosos com 60 anos, ou mais, que apresentavam o diagnóstico da doença, encaminhados pelo Hospital Regional de Coari. A média de idade dos idosos foi de 68,8 $\pm 8,35$ anos. O tempo médio de diagnóstico foi de 5,5 anos. O índice de Katz mostrou que 72\% dos idosos entrevistados eram totalmente independentes para as atividades básicas da vida diária. Foi identificado pior QV principalmente nos aspectos físicos (AF), acredita-se que a dor, a rigidez e a função articular comprometidas pela osteoartrose, provavelmente tiveram papel fundamental nesta alteração. Conclusão: Através desse estudo pode-se concluir que a articulação que mais afetou a QV dos idosos que apresentam OA, principalmente relacionada à capacidade funcional e a dor. Entre os locais mais acometidos, o joelho e a coluna foram os mais afetados.
\end{abstract}

Palavras-chave: Qualidade de vida; Osteoartrite; Pessoas com incapacidade física. 


\title{
FUNCTIONAL CAPACITY AND QUALITY OF LIFE IN ELDERLY PATIENTS WITH OSTEOARTHRITIS IN THE CITY OF COARI, AMOZONAS
}

\begin{abstract}
Introduction: Osteoarthritis (OA) is a chronic degenerative rheumatic disease with a high prevalence among the elderly that causes functional disability and high impact on the quality of life of patients. However, regional differences related to lifestyle can influence these aspects. Objective: To evaluate quality of life (QOL) and functional capacity (FC) of elderly patients with osteoarthritis in the city of Coari, Amazonas. Methods: Two questionnaires were used to measure the CF and QL, the Katz and SF36 respectively. The linear Pearson coefficient was used to measure strength of association between quantitative variables. Results: Participants were 25 elderly aged 60 years or older who had a diagnosis of the disease, referred by Regional Hospital Coari. The average age of the elderly was $68.8 \pm 8.35$ years. The median time to diagnosis was 5.5 years. The Katz showed that $72 \%$ of older respondents were totally unrelated to the basic activities of daily living. Worse QoL was identified mainly on the physical aspects $(\mathrm{AF})$, it is believed that the pain, stiffness and impaired joint function by osteoarthritis, probably played a key role in this change. Conclusion: Through this study we can conclude that the joint that most affected the QOL of elderly people who have OA, mainly related to functional capacity and pain. Among the most affected areas, knee and spine were the most affected.
\end{abstract}

Keywords: Quality of life; Osteoarthritis; People with physical disability.

\section{INTRODUÇÃO}

A osteoartrite (OA) é uma doença reumática que provoca desintegração da cartilagem articular devido às alterações bioquímicas e metabólicas. Também se pode notar o aparecimento de microfraturas, osteófitos nas bordas articulares além de cistos e esclerose no osso subcondral. Acrescentado a isso, o envelhecimento se torna um forte fator de risco, juntamente com sedentarismo, o grau de estresse na qual essa articulação foi submetida, além da pré-disposição genética que o individuo possa apresentar para o aparecimento dessa doença crônico-degenerativa. ${ }^{(1,2)}$

Os indivíduos apresentam sinais e sintomas característicos como dores noturnas, podendo ser referidas ou irradiadas, rigidez articular, diminuição da amplitude de movimento, crepitação durante a mobilização podendo chegar até mesmo na perda da função articular. $^{(3)}$

Pelo menos $10 \%$ da população idosa, no mundo, segundo a Organização Mundial de Saúde (OMS), apresenta AO, ocasionando assim limitação funcional e restrição de movimentos da maioria dos idosos que apresentam essa doença crônicodegenerativa. No Brasil, apesar do aumento da expectativa de vida, ainda não foi possível encontrar nenhum estudo relacionado à prevalência dessa doença nos idosos. ${ }^{(4)}$

Devido a essa alta prevalência de condições crônicas e incapacidade funcional encontradas na maioria dos idosos, esses indivíduos passam a ser diferenciados pelas 
suas condições de saúde, bem-estar, nas necessidades de cuidados, pois os mesmos constituem grupos com características bastante diferenciadas. Assim pode-se notar que à medida que os indivíduos envelhecem percebe-se que há uma grande variedade nos graus de incapacidade, além de uma grande diferença nas condições de saúde. ${ }^{(5)}$

A incapacidade funcional no idoso provoca uma maior vulnerabilidade e dependência da família, da comunidade ou do próprio sistema de saúde, contribuindo assim para uma redução da qualidade de vida $(\mathrm{QV})$ desses indivíduos. ${ }^{(1)}$

Essa incapacidade funcional age diretamente na QV desses indivíduos, em suas atividades de vida diária, independente da região onde a doença se instalou, levando a restrição dos movimentos, limitação, perda de força muscular, dentre outros acometimentos. ${ }^{(6)}$

O aumento da expectativa de vida possibilita cada vez mais indivíduos chegarem à velhice, porém, torna-os mais suscetíveis ao aparecimento de doenças, na medida em que envelhecem. Estudos têm demonstrado o quanto a OA pode causar limitações funcionais, diminuindo o nível de QV dos idosos, restringindo suas atividades de vida diárias. ${ }^{(2,5)}$ Conhecer o nível de QV desses idosos que apresentam OA e suas principais limitações, nas atividades da vida diária e de acordo com a região afetada, possibilita o planejamento de intervenções mais eficazes no alivio da sintomatologia e suas conseqüências funcionais.

A QV é basicamente um reconhecimento multidimensional, pois para abranger um perfil adequado de cada individuo avaliado são necessários domínios específicos. São 6 domínios: Físico; psicológico; nível de independência; relações sociais; meio ambiente; aspectos espirituais. Assim para que se tenha uma boa avaliação da QV são necessárias que sejam comparadas as condições ao longo do tempo com outros indivíduos que apresentem a mesma ou diferente condição de saúde ou de ambiente. ${ }^{(7)}$ A QV na terceira idade para ser classificada como boa ou ótima tem que envolver o mínimo de condições para a produção máxima de cada individuo, seja trabalhando, se relacionando afetivamente, entre outros. ${ }^{(8)}$

Embora muitos dados da literatura revelem o impacto sobre a $\mathrm{CF}$ e QV da OA em idosos, diferenças regionais relacionadas aos hábitos de vida, podem influenciar estes aspectos. Assim, esta pesquisa teve como objetivo avaliar a capacidade funcional e a qualidade de vida de idosos com osteoartrite, na cidade de Coari-AM.

Revista Pesquisa em Fisioterapia, Salvador, dez. 2012; 2(2): 107-120. 


\section{METODOLOGIA}

Trata-se de um estudo descritivo conduzido em idosos com OA, residentes na cidade de Coari-AM, através de questionários específicos. Participaram da pesquisa toda a demanda de idosos com OA previamente diagnosticada, que se enquadraram dentro dos critérios de inclusão e exclusão e que seguiram do Hospital Regional de Coari-AM. Para a sua realização, este trabalho foi encaminhado e apreciado pelo Comitê de Ética em Pesquisa (CEP) da Universidade Federal do Amazonas (UFAM), tendo sua aprovação sob o documento CAAE 0404.0.115.000-10.

Foram adotados como critérios de inclusão: Pacientes com OA diagnosticada em qualquer articulação; idosos com idade acima de 60 anos; ambos os gêneros. Como critérios de exclusão: Pacientes que apresentassem qualquer outro tipo de doença articular, óssea ou muscular ou outro tipo de doença reumática; apresentar prótese total ou parcial na articulação afetada pela $\mathrm{OA}$; disfunção cognitiva grave e pacientes que não concordaram com o termo de consentimento livre e esclarecido.

A Mensuração da CF foi feita através do índice de Katz, instrumento muito utilizado em estudos em gerontologia para a avaliação funcional. ${ }^{(9)} \mathrm{O}$ instrumento apresentava 6 itens a serem avaliados (Banhar-se; vestir-se; ir ao banheiro; transferência; continência; alimentar-se). A avaliação foi realizada diretamente com o terapeuta onde o mesmo atribuiu um ponto a cada atividade realizada sem ajuda. $\mathrm{O}$ valor zero foi atribuído quando não houvesse a realização da atividade. A pontuação foi combinada formando uma escala cumulativa onde posteriormente foram classificadas em números de 0 a 6 em ordem crescente de dependência fornecendo um resultado padrão exato para as respostas. ${ }^{(9,10)}$

A QV foi analisada a partir da aplicação do questionário Short Form- 36 (SF36), que se mostra muito eficiente para uma exploração completa da avaliação da qualidade de vida relacionada à saúde, sendo de fácil aplicação e compreensão. ${ }^{(10,11)}$ Foram 36 questões com variadas possibilidades de pontuação, onde oito domínios foram avaliados (capacidade funcional; aspectos físicos; dor; estado de saúde geral; vitalidade; aspectos sociais; aspectos emocionais; saúde mental) e mais uma questão comparativa entre as condições atuais de saúde e a do ano anterior à entrevista. ${ }^{(13)}$

Revista Pesquisa em Fisioterapia, Salvador, dez. 2012; 2(2): 107-120. 
A aplicação dos questionários foi individual. Após a seleção dos pacientes através dos dados das fichas ambulatoriais, no período de agosto a setembro de 2010, foi feita uma visita nas residências dos mesmos para a obtenção do consentimento para a utilização de seus dados colhidos na pesquisa. Em caso de analfabetismo do paciente foi sugerida a participação de uma pessoa de confiança do idoso para a leitura e concordância do termo de consentimento livre e esclarecido.

As informações coletadas foram arquivadas em um banco de dados desenvolvido no programa da Microsoft Office Excel $^{\circledR}$ versão 2007 e para análise estatística utilizou-se o Minitab release $14^{\circledR}$. Os dados descritivos apresentados na seção de resultados foram obtidos com a utilização de freqüências e porcentagens para os escores de Katz e variáveis categóricas além da obtenção de medidas de tendência central (média) e medida de dispersão (desvio-padrão) para os domínios do SF-36. Utilizou-se a correlação de Pearson para associação das variáveis, tomando como nível de significância estatística $\mathrm{p}<0,05$.

\section{RESULTADOS}

Foram inclusos no estudo 25 idosos com média de idade de 68,8 \pm 8,35 (60-92)

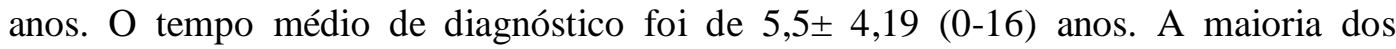
pacientes foi do gênero feminino com $60 \%$ do espaço amostral.

O índice de Katz, utilizado para a avaliação das atividades básicas da vida diária (ABVDs), mostrou que $72 \%$ dos idosos entrevistados eram totalmente independentes para essas atividades, seguido de $24 \%$ daqueles que eram dependentes apenas para uma das atividades. A Tabela 1 mostra a porcentagem obtida dos níveis de CF através do índice de Katz.

Tabela 1 - Níveis de Capacidade Funcional de acordo com o índice de Katz.

\begin{tabular}{crc}
\hline Índice de Katz & $\mathbf{N}$ & $(\boldsymbol{\%})$ \\
\hline 0 & 18 & $72 \%$ \\
1 & 6 & $24 \%$ \\
2 & 1 & $4 \%$ \\
3 & 0 & $0 \%$ \\
4 & 0 & $0 \%$ \\
5 & 0 & $0 \%$ \\
6 & 0 & $0 \%$ \\
\hline & $\mathbf{N}=\mathbf{2 5}$ & TOTAL $=\mathbf{1 0 0 \%}$ \\
\hline
\end{tabular}

Revista Pesquisa em Fisioterapia, Salvador, dez. 2012; 2(2): 107-120. 
Pode-se observar também que $48 \%$ da amostra estavam na faixa etária de 60 a 65 anos e 55,5\% dos idosos independentes para as ABVDs também se apresentam nessa faixa etária, como mostrado na Tabela 2.

Tabela 2 - Associação entre índice de Katz e Faixa etária

\begin{tabular}{|c|c|c|c|c|c|}
\hline Índice de Katz & 60 a 65 anos & 66 a 70 anos & 71 a 80 anos & 81 a 100 anos & Total geral \\
\hline 0 & $10(55,5 \%)$ & $2(11,1 \%)$ & $4(22,2 \%)$ & $2(11,1)$ & $18(100 \%)$ \\
\hline 1 & $2(33,3 \%)$ & $3(50 \%)$ & - & $1(16,6 \%)$ & $6(100 \%)$ \\
\hline 2 & - & - & $1(100 \%)$ & - & $1(100 \%)$ \\
\hline 3 & - & - & - & - & - \\
\hline 4 & - & - & - & - & - \\
\hline 5 & - & - & - & - & - \\
\hline 6 & - & - & - & - & - \\
\hline Total geral & $12(48 \%)$ & $5(20 \%)$ & $5(20 \%)$ & $3(12 \%)$ & $\mathrm{N}=25(100 \%)$ \\
\hline
\end{tabular}

$0=$ independência total; $1=$ independente para todas as funções menos uma; $2=$ Independente em quatro funções e dependente em duas; $3=$ Independente em três funções e dependente em três funções; 4= Independente em duas funções e dependente em quatro funções; 5= Independente em uma função e dependente em cinco funções; $6=$ Dependente para todas as funções.

Na avaliação da QV através do SF-36, onde o escore máximo obtido é 100 e o mínimo é zero, foram obtidos maiores escores nas variáveis, Aspecto Social (AS), Saúde Mental (SM) e Estado Geral de Saúde (EGS), com médias de, respectivamente, $72 \pm \square 26,3$ (0-100), 66,5 $\pm 24,8$ (12-100), 56,9 $\pm 18,3$ (20-92). O pior escore foi obtido no item Aspecto Físico (AF) com média de 33,2 \pm 33,1 (0-80), como mostrado na Tabela 3.

Tabela 3 - Medidas descritivas das variáveis quantitativas do SF-36

\begin{tabular}{ccccc}
\hline Variável & Média & DP & Valor Min & Valor Máx \\
\hline Capacidade Funcional & 47 & 21,5 & 10 & 85 \\
Aspectos Físicos & 33,2 & 33,1 & 0 & 80 \\
Dor & 52,5 & 20,2 & 22 & 100 \\
Estado Geral de Saúde & 56,9 & 18,3 & 20 & 92 \\
Vitalidade & 54,4 & 24,1 & 10 & 95 \\
Aspectos Sociais & 72 & 26,3 & 0 & 100 \\
Aspecto Emocional & 53,3 & 37,2 & 0 & 100 \\
Saúde Mental & 66,5 & 24,8 & 12 & 100 \\
\hline
\end{tabular}

Min, mínimo; Máx, máximo; DP, desvio padrão.

Não foi verificada uma associação entre os níveis de capacidade funcional encontrados no SF-36 com as articulações mais acometidas encontradas na pesquisa (p>0,05). A Tabela 4 mostra que, a maioria dos pacientes (56\%) apresentou como articulação acometida o joelho (gonartrose), seguido da coluna (espondiloartrose) com 
$20 \%$ da amostra. Apenas $12 \%$ da amostra apresentaram mais de uma articulação envolvida. A maioria da população investigada (44\%) obteve escore no item CF de 40 a 60 e a articulação mais acometida nesses escores foi a de joelho com $28 \%$ do total da amostra, 32\% das pessoas apresentaram os escores mais baixos da pesquisa (10 a 35), onde $20 \%$ da amostra total que apresentaram esse escore tinham gonartrose.

Tabela 4 - Associação entre variável Capacidade Funcional (SF-36) e articulação envolvida.

\begin{tabular}{ccccccc}
\hline $\begin{array}{c}\text { Escores da CF } \\
\text { (SF-36) }\end{array}$ & OA coluna & OA Joelhos & $\begin{array}{c}\text { OA } \\
\text { mãos }\end{array}$ & $\begin{array}{c}\text { OA em mais } \\
\text { de uma } \\
\text { articulação }\end{array}$ & $\begin{array}{c}\text { OA em } \\
\text { outra } \\
\text { articulação }\end{array}$ & total \\
\hline $10-35$ & $1(4 \%)$ & $5(20 \%)$ & $(0 \%)$ & $2(8 \%)$ & $(0 \%)$ & $8(32 \%)$ \\
$40-60$ & $2(8 \%)$ & $7(28 \%)$ & $1(4 \%)$ & $(0 \%)$ & $1(4 \%)$ & $11(44 \%)$ \\
$70-85$ & $2(8 \%)$ & $2(8 \%)$ & $1(4 \%)$ & $1(4 \%)$ & $(0 \%)$ & $6(24 \%)$ \\
\hline Total geral & $\mathbf{5 ( 2 0 \% )}$ & $\mathbf{1 4}(\mathbf{5 6 \%})$ & $\mathbf{2 ( 8 \% )}$ & $\mathbf{3 ( 1 2 \% )}$ & $\mathbf{1 ( 4 \% )}$ & $\mathbf{N = 2 5}$ \\
& & & & & & $(\mathbf{1 0 0 \%})$ \\
\hline
\end{tabular}

$r=-0,133 ; p=0,527$

A maioria dos idosos entrevistados possui idade de 60 e 65 anos (48\%), onde $20 \%$ do total da amostra, que se apresentaram nessa faixa etária, obtiveram os escores mais altos da pesquisa de (70 a 85), porém $16 \%$ do total, na mesma faixa etária, obteve os escores mais baixos (10-35), como mostrados na Tabela 5.

Tabela 5 - Associação entre variável Capacidade Funcional (SF-36) e faixa etária.

\begin{tabular}{cccccc}
\hline Escores da CF (SF-36) & $\begin{array}{c}\text { 60 a 65 } \\
\text { anos }\end{array}$ & $\begin{array}{c}\text { 66 a 70 } \\
\text { anos }\end{array}$ & $\begin{array}{c}\text { 71 a 80 } \\
\text { anos }\end{array}$ & $\begin{array}{c}\mathbf{8 1} \text { a 100 } \\
\text { anos }\end{array}$ & Total \\
\hline $10-35$ & $4(16 \%)$ & $1(4 \%)$ & $2(8 \%)$ & $1(4 \%)$ & $8(32 \%)$ \\
$40-60$ & $3(12 \%)$ & $3(12 \%)$ & $3(12 \%)$ & $2(8 \%)$ & $11(44 \%)$ \\
$70-85$ & $5(20 \%)$ & $1(4 \%)$ & $-(0 \%)$ & $-(0 \%)$ & $6(24 \%)$ \\
\hline Total geral & $\mathbf{1 2}(48 \%)$ & $\mathbf{5 ( 2 0 \% )}$ & $\mathbf{5 ( 2 0 \% )}$ & $\mathbf{3 ( 1 2 \% )}$ & $\mathbf{N = 2 5 ( 1 0 0 \% )}$ \\
\hline
\end{tabular}

$r=-0,164 ; p=0,433$

Na Tabela 6 pode-se observar que $52 \%$ da amostra apresentaram diagnóstico da doença na faixa de 1-5 anos, seguido de $32 \%$ na faixa de 10 anos ou mais, onde a maioria da população amostral (28\%) teve um escore de 40 a 60 na variável CF.

Tabela 6 - Associação entre variável Capacidade Funcional e tempo da doença

\begin{tabular}{cccccc}
\hline Escores da CF (SF-36) & $\begin{array}{c}\text { menos de 1 } \\
\text { ano }\end{array}$ & $\begin{array}{c}\mathbf{1} \text { a 5 } \\
\text { anos }\end{array}$ & $\begin{array}{c}\text { 6 a 9 } \\
\text { anos }\end{array}$ & $\begin{array}{c}\text { 10 anos ou } \\
\text { mais }\end{array}$ & Total \\
\hline $10-35$ & $1(4 \%)$ & $2(8 \%)$ & $2(8 \%)$ & $3(12 \%)$ & $8(32 \%)$ \\
$40-60$ & $(0 \%)$ & $7(28 \%)$ & $(0 \%)$ & $4(16 \%)$ & $11(44 \%)$ \\
$70-85$ & $1(4 \%)$ & $4(16 \%)$ & $(0 \%)$ & $1(4 \%)$ & $6(24 \%)$ \\
\hline Total geral & $\mathbf{2 ( 8 \% )}$ & $\mathbf{1 3}(\mathbf{5 2 \%})$ & $\mathbf{2 ( 8 \% )}$ & $\mathbf{8 ( 3 2 \% )}$ & $\mathbf{N = 2 5 ( 1 0 0 \% )}$ \\
\hline
\end{tabular}

$\mathrm{r}=-0,322 ; \mathrm{p}=0,117$ 
De acordo com a população que apresenta 10 anos ou mais a doença, $12 \%$ apresentaram escores muito baixos (10 a 35), ou seja, a maioria dos indivíduos que obteve esse escore convivia 10 anos ou mais com a doença, como mostrado na Tabela 7.

Tabela 7 - Associação entre variável Dor e articulação envolvida

\begin{tabular}{ccccccc}
\hline $\begin{array}{c}\text { Escores Dor } \\
\text { (SF-36) }\end{array}$ & $\begin{array}{c}\text { OA } \\
\text { coluna }\end{array}$ & $\begin{array}{c}\text { OA } \\
\text { joelhos }\end{array}$ & $\begin{array}{c}\text { OA } \\
\text { mãos }\end{array}$ & $\begin{array}{c}\text { OA mais de } \\
\text { uma } \\
\text { articulação }\end{array}$ & $\begin{array}{c}\text { OA em } \\
\text { outra } \\
\text { articulação }\end{array}$ & total \\
\hline $22-41$ & $3(12 \%)$ & $2(8 \%)$ & $(0 \%)$ & $3(12 \%)$ & $(0 \%)$ & $8(32 \%)$ \\
$42-54$ & $(0 \%)$ & $5(20 \%)$ & $1(4 \%)$ & $(0 \%)$ & $(0 \%)$ & $6(24 \%)$ \\
$62-100$ & $2(8 \%)$ & $7(28 \%)$ & $1(4 \%)$ & $(0 \%)$ & $1(4 \%)$ & $11(44 \%)$ \\
\hline Total geral & $\mathbf{5 ( 2 0 \% )}$ & $\mathbf{1 4 ( 5 6 \% )}$ & $\mathbf{2 ( 8 \% )}$ & $\mathbf{3 ( 1 2 \% )}$ & $\mathbf{1 ( 4 \% )}$ & $\begin{array}{c}\mathbf{N = 2 5} \\
(\mathbf{1 0 0 \%})\end{array}$ \\
\hline $\mathrm{r}=0,068 ; \mathrm{p}=0,748$ & & & & & &
\end{tabular}

Os pacientes que obtiveram menores escores na avaliação do item dor foram os acometido por espondiloartrose (12\%), igualmente aos que apresentaram mais de uma articulação acometida (12\%). Os melhores escores (62-100) tinham $28 \%$ de gonartrose. De acordo com a tabela 8 pôde-se perceber que a maioria da população de 60 a 65 obtiveram na variável dor os escores mais altos (28\%) e os mais baixos ficaram com a população de faixa etária de 66 a 70 (16\%).

Tabela 8 - Associação entre variável Dor e faixa etária.

\begin{tabular}{cccccc}
\hline Escores Dor (SF-36) & $\begin{array}{c}\mathbf{6 0} \text { a } 65 \\
\text { anos }\end{array}$ & $\begin{array}{c}\mathbf{6 6} \text { a 70 } \\
\text { anos }\end{array}$ & $\begin{array}{c}\mathbf{7 1} \text { a 80 } \\
\text { anos }\end{array}$ & $\begin{array}{c}\mathbf{8 1} \text { a 100 } \\
\text { anos }\end{array}$ & Total \\
\hline $22-41$ & $2(8 \%)$ & $4(16 \%)$ & $2(8 \%)$ & $(0 \%)$ & $8(32 \%)$ \\
$42-54$ & $3(12 \%)$ & $(0 \%)$ & $2(8 \%)$ & $1(4 \%)$ & $6(24 \%)$ \\
$62-100$ & $7(28 \%)$ & $1(4 \%)$ & $1(4 \%)$ & $2(8 \%)$ & $11(44 \%)$ \\
\hline Total geral & $\mathbf{1 2 ( 4 8 \% )}$ & $\mathbf{5 ( 2 0 \% )}$ & $\mathbf{5 ( 2 0 \% )}$ & $\mathbf{3 ( 1 2 \% )}$ & $\mathbf{N = 2 5 ( 1 0 0 \% )}$ \\
\hline$r=-0,107 ; p=0,609$ & & & & &
\end{tabular}

$\mathrm{r}=-0,107 ; \mathrm{p}=0,609$

A maioria dos idosos (16\%) que obtiveram menor escore na variável dor eram os que apresentavam de 1-5 anos de diagnóstico da doença, seguido dos que apresentavam 10 anos ou mais (12\%). Os escores mais altos (62-100) foram obtidos pelos pacientes que têm de um a cinco anos, de diagnóstico, em um total de 32\% (Tabela 9). 
Tabela 9 - Associação entre variável Dor e tempo da doença.

\begin{tabular}{cccccc}
\hline Escores Dor (SF-36) & $\begin{array}{c}\text { Menos } \\
\text { de } \mathbf{1} \text { ano }\end{array}$ & $\mathbf{1}$ a 5 anos & $\begin{array}{c}\text { 6 a 9 } \\
\text { anos }\end{array}$ & $\begin{array}{c}\text { 10 ou mais } \\
\text { anos }\end{array}$ & Total \\
\hline $22-41$ & $(0 \%)$ & $4(16 \%)$ & $1(4 \%)$ & $3(12 \%)$ & $8(32 \%)$ \\
$42-54$ & $(0 \%)$ & $1(4 \%)$ & $1(4 \%)$ & $4(16 \%)$ & $6(24 \%)$ \\
$62-100$ & $2(8 \%)$ & $8(32 \%)$ & $(0 \%)$ & $1(4 \%)$ & $11(44 \%)$ \\
\hline Total geral & $\mathbf{2 ( 8 \% )}$ & $\mathbf{1 3}(\mathbf{5 2 \%})$ & $\mathbf{2 ( 8 \% )}$ & $\mathbf{8 ( 3 2 \% )}$ & $\mathbf{N = 2 5 ( 1 0 0 \% )}$ \\
\hline
\end{tabular}

$r=-0,450 ; p=0,024$

Não houve resultados significativos entre as associações, com exceção da associação entre dor e tempo da doença que apresentou uma correlação moderada $(r=-$ $0,450 ; p=0,024)$.

\section{DISCUSSÃO}

A avaliação da QV de pacientes com OA através de questionários específicos mostrou o quanto a avaliação da $\mathrm{CF}$ de idosos é essencial para a escolha de uma intervenção e monitorização do estado cinético-funcional dos idosos. ${ }^{(1,14)}$

Os resultados do índice de Katz mostraram que para as ABVDs a maioria dos idosos foi totalmente independente, resultado esse que não condiz com a média baixa apresentada pelo domínio "CF" do SF-36, pois apesar dos dois avaliarem a CF o SF-36 enfatiza as atividades instrumentais da vida diária (AIVDs), que indicam a capacidade do indivíduo de levar uma vida independente dentro da comunidade onde vive e inclui a capacidade para a realização de atividades vigorosas, moderadas, deambular por distâncias razoáveis, subir e descer escadas, preparar refeições, realizar compras, utilizar transporte, cuidar da casa, utilizar telefone, administrar as próprias finanças, tomar seus medicamentos. ${ }^{(9)}$

Foi identificado pior QV principalmente nos aspectos físicos. Estes aspectos são avaliados através dos domínios CF, AF, Dor, EGS. A dor, a rigidez e a função articular comprometidas pela OA, provavelmente tiveram papel fundamental nesta alteração, uma vez que as médias mais baixas obtidas foram nos domínios CF, AF e Dor.

A dor crônica é hoje conceituada como um fenômeno de difícil entendimento e que envolve diversos fatores, como aspectos orgânicos e psicossociais, e interfere diretamente na QV dos idosos. A dor confronta o idoso com sua fragilidade e ameaça 
sua segurança, algumas vezes, impedindo a realização das AVDs, consome sua renda e esgota de modo físico e psíquico tanto a pessoa como a família ou o cuidador. ${ }^{(15)}$

Um fato importante nesse estudo é que apesar da possível limitação funcional dos pacientes, os aspectos sociais e a saúde mental que foram avaliados não estão diretamente ligados a uma piora na qualidade da QV. As médias obtidas nesses itens foram superiores aqueles relacionados aos demais aspectos. Entretanto a disfunção, dor e degradação articular podem interferir diretamente na QV por levarem na maioria das vezes os pacientes à depressão e ao isolamento social onde teriam mais chances de apresentar problemas mentais quando comparados com indivíduos sem OA. ${ }^{(16,17)}$

Através do cruzamento entre o domínio CF (SF-36) e articulação envolvida dos pacientes pode-se perceber que a articulação mais acometida foi a de joelho (gonartrose), principalmente entre os escores mais baixos desse item. Mostrando assim que a articulação do joelho caracteriza-se como uma das principais áreas de acometimento da OA e está presente em cerca de $6 \%$ da população adulta acima de 30 anos, onde a sua prevalência aumenta para $10 \%$ em pessoas com mais de 55 anos de idade. ${ }^{(18)} \mathrm{Na}$ gonoartrose, observa-se precocemente diminuição de força de flexores e extensores de joelho que estão relacionadas à diminuição da $\mathrm{CF}$ e a habilidade em realizar atividades como subir escadas, levantar, sentar e caminhar, itens explorados na avaliação de CF (do SF-36). ${ }^{(19)}$

Também se verificou que a CF varia muito entre os grupos diferentes, sendo que os sintomas da gonartrose podem fazer com que os indivíduos evitem atividades que provoquem ou intensifiquem a dor, o que acarreta a piora do condicionamento físico e a uma maior fraqueza muscular. ${ }^{(18)}$

Na presente análise a idade também se apresentou como fator importante, já que a maioria dos pacientes com OA encontrava-se na faixa etária de 60 a 65 anos, totalizando $48 \%$ idosos entrevistados. Desses idosos, com essa faixa etária, a maioria obteve escores altos de CF (SF-36), porém esse resultado não poderia afirmar que quanto maior a faixa etária, menores os níveis de capacidade funcional, pois uma porcentagem significativa de entrevistados obteve escores baixíssimos estando na mesma faixa etária. Apesar do desempenho funcional, dos indivíduos, declinar progressivamente após a terceira década de vida, devido às alterações fisiológicas do envelhecimento, muitas vezes trazem limitações funcionais apresentando maior 
repercussão nas atividades da vida diária de um idoso, dependendo da articulação envolvida na doença. ${ }^{(14)}$

A maioria dos idosos entrevistados tem de 1-5 anos de diagnóstico da doença, mas não obtiveram escores tão baixos, em CF, comparados aos idosos que apresentam o diagnóstico da doença por dez anos ou mais. Isso se deve a limitação do movimento que vai gerar atrofia muscular, incapacidade funcional, diminuição da força muscular, levando a um ciclo vicioso, que por sua vez, leva a piora da QV do paciente. O grau de mobilidade pode apresentar-se diminuído pela fibrose capsular, osteofitose, irregularidade das superfícies articulares, impacto dos corpos livres e também pela própria dor. A atrofia muscular está presente secundariamente ao desuso, pois os músculos que atuam sobre as grandes articulações tornam-se atróficos. Em um estágio final ou avançado da osteoartrose pode haver uma fixação da articulação em posição defeituosa causando deformidades. ${ }^{(20)}$

Apesar da OA de joelho ser a mais encontrada entre os idosos, os dados colhidos mostram que a OA de coluna e os pacientes que apresentam mais de uma articulação acometida têm os menores escores para dor, conseqüentemente apresentam uma menor CF. A dor e as incapacidades prejudicam a mobilidade, resultando em desvantagem e piora da integração social. Idosos vivendo com OA relatam que eles restringem sua mobilidade, principalmente quando a dor está exacerbada, deixando de realizar suas atividades sociais rotineiras. ${ }^{(19,21)}$

Pôde-se perceber que, os melhores escores para dor foram dos idosos que estão na faixa etária de 60 a 65 anos, estudos apontam para a influência da idade na OA, mostrando que a idade influencia na gravidade da doença, principalmente em mulheres. ${ }^{(19,18)}$

De acordo com a associação do domínio dor e o tempo da doença pode-se perceber que a maioria dos idosos entrevistados com 10 anos ou mais de diagnóstico da doença apresentaram a dor como um fator bastante limitante que influencia diretamente na QV. Através do teste estatístico essa correlação foi dada como significativa, ou seja, para essa amostra quanto mais tempo a pessoa estiver com essa doença crônicodegenerativa mais dores apresentarão. A causa da dor também varia dependendo do estágio da $\mathrm{OA}$. Alguns casos têm episódios intermitentes de dor devido à sinovite. $\mathrm{O}$ 
aumento da pressão intra-óssea devido à congestão vascular do osso subcondral é freqüente, assim como fibrose capsular, contratura articular e fadiga muscular. ${ }^{(22)}$

\section{CONCLUSÃO}

Através desse estudo pode-se concluir que a articulação que mais afetou a QV dos idosos que apresentam OA, principalmente relacionada à capacidade funcional e a dor, foi a de joelho, seguida da articulação da coluna. A redução da CF, dos aspectos físicos e os altos níveis de dor foram fatores que influenciaram diretamente em um pior nível de QV, dos idosos, na amostra estudada.

Manter a CF implica indiscutivelmente na QV dos idosos, por estar relacionada com a capacidade de se ocupar com o trabalho, "ser útil", até idades mais avançadas, ou pelo menos participar de atividades de seu agrado. A partir disso torna-se fundamental a implantação de políticas preventivas e de reabilitação efetivas, para que haja uma diminuição dos fatores de risco que possam levar o idoso à incapacidade funcional.

Para a manutenção da CF é necessário que se tenha um programa de atividade física regular além da implantação de um estilo de vida ativo, para que se tenha uma promoção da saúde e da QV durante o envelhecimento. A atividade física deve ser estimulada como forma de manter a independência funcional, além de melhorar a mobilidade e a QV.

\section{REFERÊNCIAS}

1. Tamegushi AS et al. Capacidade Funcional de Idosos com osteoartrite de joelho e quadril. Revista Espaço para a Saúde. 2008; 2: p.08-16.

2. Marques AP et al. A fisioterapia na osteoartrose: uma revisão de literatura. Rev. bras. reumatol. 1998; 38(2).

3. Felice JC et al. Elementos Básicos no diagnóstico da osteoartrose. Temas de Reumatologia Clínica. 2002; 3: 68-81.

4. Matos DR et al. Qualidade de vida e envelhecimento: questões específicas sobre a osteoartrose. Psicol. estud. 2009; 14(3). 
5. Alves LC et al. Perfis de saúde dos idosos no Brasil: análise da pesquisa nacional por amostra de domicílios de 2003 utilizando o método grade of membership. Cad. saúde pública. 2008; 24(3).

6. Patrizzi LJ et al. Análise pré e pós operatória da capacidade funcional e qualidade de vida de pacientes portadores de artrose de quadril submetidos a artroplastia total. Rev. bras. reumatol. 2004; 44:185-91.

7. Rebelatto JR, Morelli JGS. Fisioterapia Geriátrica: a prática da assistência ao idoso. $2^{a}$ ed. São Paulo: Manole; 2007.

8. Torres $\mathrm{GV}$ et al. Qualidade de vida e fatores associados em idosos dependentes em uma cidade do interior do Nordeste. J. bras. psiquiatr. 2009; 58(1): 2009.

9. Duarte YAO, Andrade CL, Lebrão ML. O Índex de Katz na avaliação da funcionalidade dos idosos. Rev. Esc. Enferm. USP. 2007; 41(2): 317-25.

10. Sullivan SB, Schmitz TJ. Fisioterapia Avaliação e Tratamento. $4^{\mathrm{a}}$ ed. São Paulo: Manole; 2004.

11. Maciel ACC, Guerra RO. Influência dos fatores biopsicossociais sobre a capacidade funcional de idosos residentes no nordeste do Brasil. Rev. bras. epidemiol. 2007; 10(2).

12. Cruz KC, Diogo MJ. Avaliação da Capacidade Funcional em Idosos com Acidente Vascular Encefálico. Acta Paul Enferm, 2009; 22(5): 666-72.

13. Ciconelli RM et al. Tradução para a língua portuguesa e validação do questionário genérico de avaliação de qualidade de vida SF-36. Rev. bras. reumatol. 199; 39(3):14350 .

14. Schneider RH, Marcolin D, Dalacorte RR. Avaliação funcional de idosos. Sci. med. 2008; 18(1): 4-9.

15. Celich KLS, Galon C. Dor crônica em idosos e sua influência nas atividades da vida diária e convivência social. Rev. bras. geriatr. gerontol. 2009; 12(3).

16. Krasnoutsky S, Samuels J, Abramson SB. Osteoarthritis in 2007. Bulletin of the NYU Hospital for Joint Diseases. 2007; 65(3): 222-8.

17. Cook C, Pietrobon R, Hegedus E. Osteoarthritis and the impact to quality of life health indicators. Rheumatol Int. 2007; 27: 315-21.

18. Posis RG, Massabki OS, Kairalla M. Osteoartrite: avaliação clínica e epidemiológica de pacientes idosos em instituição de longa permanência. Rev. Soc. Bras. Clín. Méd. 2010; 8(2):101-8. 
19. Melo SIL et al. Avaliação da força muscular de flexores e extensores de joelho em indivíduos com e sem osteoartrose. Rev. bras. cineantropom. desempenho hum. 2008; 10(4): 335-340.

20. Golding DN. Reumatologia em medicina e reabilitação. São Paulo: Atheneu, 2001. p.334.

21. Alexandre TS, Cordeiro RC, Ramos, RL. Fatores associados à qualidade de vida em idosos com osteoartrite de joelho. Fisioter. pesqui. 2008; 15(4).

22. Solomon L. Clinical features of osteoarthritis. In: Kelley WN, Harris ED, Ruddy S, Sledge CB. Textbook of Rheumatology. $5^{\text {a }}$ ed. Philadelphia: W.B. Saunders; 1997, p.1383-1393. 